\title{
Certain Results Comprising the Weighted Chebyshev Function Using Pathway Fractional Integrals
}

\author{
Aditya Mani Mishra ${ }^{1}$, Dumitru Baleanu ${ }^{2,3}$ [D, Fairouz Tchier ${ }^{4}\left(\mathbb{D}\right.$ and Sunil Dutt Purohit ${ }^{1, *(\mathbb{C})}$ \\ 1 Department of HEAS (Mathematics), Rajasthan Technical University, Kota 324010, India; \\ adm.nita@gmail.com \\ 2 Department of Mathematics, Cankaya University, Cankaya, Ankara 06430, Turkey; dumitru@cankaya.edu.tr \\ 3 Institute of Space Sciences, Magurele-Bucharest R7690, Romania \\ 4 Department of Mathematics, King Saud University, Riyadh 11451, Saudi Arabia; ftchier@ksu.edu.sa \\ * Correspondence: sunil_a_purohit@yahoo.com
}

Received: 30 June 2019; Accepted: 16 September 2019; Published: 25 September 2019

check for updates

\begin{abstract}
An analogous version of Chebyshev inequality, associated with the weighted function, has been established using the pathway fractional integral operators. The result is a generalization of the Chebyshev inequality in fractional integral operators. We deduce the left sided Riemann Liouville version and the Laplace version of the same identity. Our main deduction will provide noted results for an appropriate change to the Pathway fractional integral parameter and the degree of the fractional operator.
\end{abstract}

Keywords: Riemann Liouville fractional integral operator; pathway fractional order integral operator; Chebyshev functional

\section{Introduction and Preliminaries}

Fractional calculus has been broadly used in various disciplines of engineering and science as a mathematical model. Nowadays, fractional order (arbitrary order) calculus is more realistic than classical models of integer order for the memory description and hereditary rules of various physical problems and actions. Some important generalised fractional integral operators include the Hadamard operator, Erdélyi-Kober operators, the Saigo operator, the Gaussian hypergeometric operator, the Marichev-Saigo-Maeda fractional integral operator, etc.; out of those, the Riemann Liouville fractional integral operator has been widely used by researchers in theory as well as applications. For additional details about fractional calculus operators and their utility, one may refer to the treatises by Miller and Ross [1], Samko et al. [2], Kiryakova [3] and Baleanu et al. [4]. The pathway fractional integral operator is a generalised Riemann Liouville fractional integral operator in higher dimensions. Indeed, the pathway fractional integral operator has been used to define certain probability density functions and has interesting applications in statistics (also see [5-7]).

Let $\lambda$ be an arbitrary real number preferably less than 1 . Let a class of conformal functions:

$$
C_{\lambda}^{(k)}=\left\{f(t)=t^{p} \tilde{f}(t) ; p>\lambda, \tilde{f} \in C^{(k)}([\alpha, \beta])\right\}
$$

be a Banach space for our consideration for different non-negative values of $k$. The space we have chosen for $k=0[3]$ is induced by the norm:

$$
\|f\|_{r}=\left[\int_{\alpha}^{\beta}|f(t)|^{r} d t\right]^{\frac{1}{r}}<\infty .
$$


Suppose $\phi(t) \in C_{\lambda}^{(k)}$ for $k=0$ and $v \in \mathbb{C}$, which has a positive real part, and $a$ is positive. The pathway fractional integral operator is defined as ([5]):

$$
P_{0^{+}}^{(v, \lambda)}(\phi(t))=\int_{0}^{\frac{t}{a(1-\lambda)}} t^{v}\left[1-\frac{a(1-\lambda) u}{t}\right]^{v /(1-\lambda)} \phi(u) d u
$$

We call $\lambda$ the pathway parameter and $v$ the order of the integral operator. While $\lambda$ converges to 1 from the left side, the operator gets the form:

$$
P_{0^{+}}^{(v, 1)}(\phi(t))=\int_{0}^{t / a(1-\lambda)} t^{v} e^{\frac{-a v u}{t}} \phi(u) d u=t^{v} \mathfrak{L}\left[\frac{a v}{t}\right] .
$$

Meaning thereby that the fractional integral operator of pathway type reduces to the Laplace transform with the factor $(a v / t)$ for a particular value of $\lambda$.

The pathway fractional order integral operator transformed into a left-sided fractional order integral operator of Riemann-Liouville type, i.e., $I_{0+}^{v}(\phi(t))$ for a particular value of $\lambda=0$ and $a=1$ :

$$
\int_{0}^{t}(t-u)^{v-1} \phi(u) d u=\Gamma(v) I_{0+}^{v}(\phi(t))
$$

by replacing $v$ to $v-1$. The reader may refer to the papers of Mathai and Haubold [6,7], Nair [8] and Nisar et al. [9] for more details on pathway operators.

Further, an operator $T$ defined from $X$ and $\phi, \psi \in X$, called the weighted version of the Chebyshev functional, is:

$$
T(\phi, \psi, p)=\int_{\alpha}^{\beta} p(t) d t \int_{\alpha}^{\beta} \phi(t) \psi(t) p(t) d t-\int_{\alpha}^{\beta} \phi(t) p(t) d t \int_{\alpha}^{\beta} \psi(t) p(t) d t,
$$

where $p(t)$ is the weighted function with positive values. A result has been established by Dragomir [10] related to Equation (4), given by:

$$
2|T(\phi, \psi, p)| \leq|| \phi^{\prime}\left|\left\|_{r}|| \psi^{\prime}\right\|_{s}\left[\int_{\alpha}^{\beta} \int_{\alpha}^{\beta}|u-v| p(u) p(v) d u d v\right] .\right.
$$

Here, $\phi, \psi$ are derivable functions and $\phi^{\prime} \in L_{r}([\alpha, \beta]), \psi^{\prime} \in L_{s}([\alpha, \beta])$ with the condition that $r^{-1}+s^{-1}=1$. Recently, by using fractional integral operators, several extensions of the classical inequalities, including Equation (5), have been studied by many authors, see [11-24] and the references therein. We attempt to generate the inequality of Equation (5) by making use of the fractional integral operator of pathway type. So, in this article our prime intention is to provide an analogous version of the Chebyshev inequality by means of pathway integral operators of fractional orders. The result is a generalization of the Chebyshev inequality in fractional integral operators. We also deduce the left-sided Riemann-Liouville version and Laplace version of the same inequality.

The article has been organised as follows: First we prove a generalised version of the vital result and then state a specific but useful theorem. In Section 3, we derive certain specific results using these two theorems in the form of corollaries.

\section{Main Theorems}

We state the subsequent results: 
Theorem 1. Let $\phi(t), \psi(t) \in C_{\lambda}^{(k)}$ for $k=0$ and $v \in \mathbb{C}$, such that the real part of $v$ is positive and $\phi^{\prime} \in L_{r}([0, \infty))$ and $\psi^{\prime} \in L_{s}([0, \infty))$ have the condition $r^{-1}+s^{-1}=1$ (when $\left.r>1\right)$. $p$ is a positive function and we choose $a>0$ and $\lambda<1$. Therefore, for every $t \in[0, \infty)$ :

$$
\begin{array}{r}
\mid P_{0^{+}}^{(v, \lambda)}(\phi(t) \psi(t) p(t)) P_{0^{+}}^{\left(v^{\prime}, \lambda^{\prime}\right)}(p(t))-P_{0^{+}}^{(v, \lambda)}(\psi(t) p(t)) P_{0^{+}}^{\left(v^{\prime}, \lambda^{\prime}\right)}(\phi(t) p(t)) \\
-P_{0^{+}}^{(v, \lambda)}(\phi(t) p(t)) P_{0^{+}}^{\left(v^{\prime}, \lambda^{\prime}\right)}(\psi(t) p(t))+P_{0^{+}}^{(v, \lambda)}(p(t)) P_{0^{+}}^{\left(v^{\prime}, \lambda^{\prime}\right)}(\phi(t) \psi(t) p(t)) \mid \\
\leq\left\|\phi^{\prime}\right\|\left\|_{r}\right\| \psi^{\prime} \|_{s} t \int_{0}^{\overline{a(1-\lambda)}} \int_{0}^{\overline{a\left(1-\lambda^{\prime}\right)}} F(t, u) G(t, v) p(u) p(v) d v d u \\
\leq\left\|\phi^{\prime} \mid\right\|_{r}\left\|\psi^{\prime}\right\|_{s} t P_{0^{+}}^{(v, \lambda)}(p(t)) P_{0^{+}}^{\left(v^{\prime}, \lambda^{\prime}\right)}(p(t)),
\end{array}
$$

where $P_{0^{+}}^{(v, \lambda)}(\phi(t))$ and $P_{0^{+}}^{\left(v^{\prime}, \lambda^{\prime}\right)}(\psi(t))$ are pathway operators in $C_{\lambda}^{(k)}$ space and $H(t, v), F(t, u)$ and $G(t, v)$ are functions in two-dimensional space defined as follows:

$$
\begin{gathered}
H(u, v)=(\phi(u)-\phi(v))(\psi(u)-\psi(v)) \\
F(t, u)=t^{v}\left[1-\frac{a(1-\lambda) u}{t}\right]^{v /(1-\lambda)}
\end{gathered}
$$

where $u \in[0, t]$ when $t \in[0, \infty)$ and:

$$
G(t, v)=t^{v^{\prime}}\left[1-\frac{a\left(1-\lambda^{\prime}\right) v}{t}\right]^{v^{\prime} /\left(1-\lambda^{\prime}\right)} .
$$

Proof. Multiplying $H(u, v)$, defined in Equation (7), by $F(t, u) p(u)$ and integrating $u$ over 0 to $t / a(1-\lambda)$ :

$$
\begin{aligned}
& \int_{0}^{\frac{t}{a(1-\lambda)}} H(t, v) F(t, u) p(u) d u=\int_{0}^{t / a(1-\lambda)} t^{v}\left[1-\frac{a(1-\lambda) u}{t}\right]^{v /(1-\lambda)}[\phi(u) \psi(u) p(u) d u \\
& -\phi(v) \psi(u) p(u) d u-\phi(u) \psi(v) p(u) d u+\phi(v) \psi(v) p(u) d u] .
\end{aligned}
$$

This implies:

$$
\begin{array}{r}
\int_{0}^{\frac{t}{a(1-\lambda)}} H(t, v) F(t, u) p(u) d u=P_{0^{+}}^{(v, \lambda)}(\phi(t) \psi(t) p(t)) \\
-\phi(v) P_{0^{+}}^{(v, \lambda)}(\psi(t) p(t))-\psi(v) P_{0^{+}}^{(v, \lambda)}(\phi(t) p(t))+\phi(v) \psi(v) P_{0^{+}}^{(v, \lambda)}(p(t)) .
\end{array}
$$


Now by multiplying Equation (11) by $G(t, v) p(v)$ and integrating with respect to $v$ between 0 and $v^{\prime} / a\left(1-\lambda^{\prime}\right)$, we get:

$$
\begin{aligned}
& \int_{0}^{\frac{t}{a(1-\lambda)}} \int_{0}^{\frac{t}{a\left(1-\lambda^{\prime}\right)}} H(u, v) F(t, u) G(t, v) p(u) p(v) d v d u \\
& =\left(\int_{0}^{\frac{t}{a(1-\lambda)}} t^{v}\left[1-\frac{a(1-\lambda) u}{t}\right]^{v /(1-\lambda)} \phi(u) \psi(u) p(u) d u\right) \\
& \times\left(\int_{0}^{\frac{t}{a\left(1-\lambda^{\prime}\right)}} t^{v^{\prime}}\left[1-\frac{a\left(1-\lambda^{\prime}\right) v}{t}\right]^{v^{\prime} /\left(1-\lambda^{\prime}\right)} p(v) d v\right) \\
& -\left(\int_{0}^{\frac{t}{a(1-\lambda)}} t^{v}\left[1-\frac{a(1-\lambda) u}{t}\right]^{v /(1-\lambda)} \psi(u) p(u) d u\right) \\
& \times\left(\int_{0}^{\frac{t}{a(1-\lambda \prime)}} t^{v \prime}\left[1-\frac{a(1-\lambda \prime) v}{t}\right]^{v \prime /(1-\lambda \prime)} \phi(v) p(v) d v\right) \\
& -\left(\int_{0}^{\frac{t}{a(1-\lambda)}} t^{v}\left[1-\frac{a(1-\lambda) u}{t}\right]^{v /(1-\lambda)} \phi(u) p(u) d u\right) \\
& \times\left(\int_{0}^{\frac{t}{a(1-\lambda /)}} t^{v},\left[1-\frac{a(1-\lambda \prime) v}{t}\right]^{v^{\prime} /(1-\lambda \prime)} \psi(v) p(v) d v\right) \\
& +\left(\int_{0}^{\frac{t}{a(1-\lambda)}} t^{v}\left[1-\frac{a(1-\lambda) u}{t}\right]^{v /(1-\lambda)} p(u) d u\right) \\
& \times\left(\int_{0}^{\frac{t}{a(1-\lambda \prime)}} t^{v \prime}\left[1-\frac{a(1-\lambda \prime) v}{t}\right]^{\prime \prime \prime /(1-\lambda \prime)} \phi(v) \psi(v) p(v) d v\right)
\end{aligned}
$$

This implies:

$$
\begin{array}{r}
\int_{0}^{\frac{t}{a(1-\lambda)} \int_{0}^{\frac{t}{a\left(1-\lambda^{\prime}\right)}} H(u, v)} F(t, u) G(t, v) p(u) p(v) d v d u=P_{0^{+}}^{(v, \lambda)}(\phi(t) \psi(t) p(t)) \\
\times P_{0^{+}}^{\left(v^{\prime}, \lambda^{\prime}\right)}(p(t))-P_{0^{+}}^{(v, \lambda)}(\psi(t) p(t)) P_{0^{+}}^{\left(v^{\prime}, \lambda^{\prime}\right)}(\phi(t) p(t)) \\
-P_{0^{+}}^{(v, \lambda)}(\phi(t) p(t)) P_{0^{+}}^{\left(v^{\prime}, \lambda^{\prime}\right)}(\psi(t) p(t))+P_{0^{+}}^{(v, \lambda)}(p(t)) P_{0^{+}}^{\left(v^{\prime}, \lambda^{\prime}\right)}(\phi(t) \psi(t) p(t)) .
\end{array}
$$

Now, by the Hölder inequality:

$$
|H(u, v)| \leq\left.\left.\left.\left.|u-v|\left|\int_{u}^{v}\right| \phi^{\prime}\right|^{r} d x\right|^{r^{-1}}\left|\int_{u}^{v}\right| \psi^{\prime}\right|^{s} d t\right|^{s^{-1}},
$$

with $r^{-1}+s^{-1}=1$. We apply the Hölder inequality on the left side of Equation (13). The left side of Equation (13) converts to:

$$
\begin{array}{r}
\int_{0}^{\frac{t}{a(1-\lambda)}} \int_{0}^{\frac{t}{a\left(1-\lambda^{\prime}\right)}} H(u, v) F(t, u) G(t, v) p(u) p(v) d v d u \leq \int_{0}^{\frac{t}{a(1-\lambda)}} \int_{0}^{\frac{t}{a\left(1-\lambda^{\prime}\right)}} F(t, u) G(t, v) p(u) p(v) \\
\times\left.\left.\left.\left.|u-v|\left|\int_{u}^{v}\right| \phi^{\prime}\right|^{r} d x\right|^{r^{-1}}\left|\int_{u}^{v}\right| \psi^{\prime}\right|^{s} d t\right|^{s^{-1}} d v d u .
\end{array}
$$

Now, we will use the Hölder inequality, but in the form of:

$$
\left|\int_{u}^{v} \int_{u}^{v} f(x) g(t) d x d t\right| \leq\left.\left.\left.\left.\left|\int_{u}^{v} \int_{u}^{v}\right| f(x)\right|^{r} d x d t\right|^{r^{-1}}\left|\int_{u}^{v} \int_{u}^{v}\right| g(t)\right|^{s} d x d t\right|^{s^{-1}}
$$


to get:

$$
\begin{array}{r}
\int_{0}^{\frac{t}{a(1-\lambda)}} \int_{0}^{\frac{t}{a\left(1-\lambda^{\prime}\right)}} H(u, v) F(t, u) G(t, v) p(u) p(v) d v d u \\
\leq\left[\left.\int_{0}^{\frac{t}{a(1-\lambda)}} \int_{0}^{\frac{t}{a\left(1-\lambda^{\prime}\right)}} F(t, u) G(t, v) p(u) p(v)|u-v|\left|\int_{u}^{v}\right| \phi^{\prime}(x)\right|^{r} d x \mid d u d v\right]^{r^{-1}} \\
\times\left[\left.\int_{0}^{\frac{t}{a(1-\lambda)}} \int_{0}^{\frac{t}{a\left(1-\lambda^{\prime}\right)}} F(t, u) G(t, v) p(u) p(v)|u-v|\left|\int_{u}^{v}\right| \psi^{\prime}(t)\right|^{s} d t \mid d u d v\right]^{s^{-1}} \\
=\left[\left\|\phi^{\prime}\right\|_{r}^{r} \int_{0}^{\frac{t}{a(1-\lambda)}} \int_{0}^{\frac{t}{a\left(1-\lambda^{\prime}\right)}} F(t, u) G(t, v) p(u) p(v)|u-v| d u d v\right]^{r^{-1}} \\
\times\left[\left\|\psi^{\prime}\right\|_{s}^{s} \int_{0}^{\frac{t}{a(1-\lambda)}} \int_{0}^{\frac{t}{a\left(1-\lambda^{\prime}\right)}} F(t, u) G(t, v) p(u) p(v)|u-v| d u d v\right]^{s^{-1}} \\
\leq|| \phi^{\prime}\left|\left\|_{r}|| \psi^{\prime}\left|\|_{s}\right| \int_{0}^{\frac{t}{a(1-\lambda)}} \int_{0}^{\frac{t}{a\left(1-\lambda^{\prime}\right)}} F(t, u) G(t, v) p(u) p(v)|u-v| d v d u \mid\right.\right.
\end{array}
$$

We look at this inequality:

$$
\begin{array}{r}
\mid P_{0^{+}}^{(v, \lambda)}(\phi(t) \psi(t) p(t)) P_{0^{+}}^{\left(v^{\prime}, \lambda^{\prime}\right)}(p(t))-P_{0^{+}}^{(v, \lambda)}(\psi(t) p(t)) P_{0^{+}}^{\left(v^{\prime}, \lambda^{\prime}\right)}(\phi(t) p(t)) \\
-P_{0^{+}}^{(v, \lambda)}(\phi(t) p(t)) P_{0^{+}}^{\left(v^{\prime}, \lambda^{\prime}\right)}(\psi(t) p(t))+P_{0^{+}}^{(v, \lambda)}(p(t)) P_{0^{+}}^{\left(v^{\prime}, \lambda^{\prime}\right)}(\phi(t) \psi(t) p(t)) \mid \\
\quad \leq\left\|\phi^{\prime}\right\|\left\|_{r}|| \psi^{\prime}\right\|_{s} \int_{0}^{\overline{a(1-\lambda)}} \int_{0}^{\frac{t}{a\left(1-\lambda^{\prime}\right)}} F(t, u) G(t, v) p(u) p(v)|u-v| d v d u
\end{array}
$$

since $|u-v|<t:$

$$
\begin{array}{r}
\mid P_{0^{+}}^{(v, \lambda)}(\phi(t) \psi(t) p(t)) P_{0^{+}}^{\left(v^{\prime}, \lambda^{\prime}\right)}(p(t))-P_{0^{+}}^{(v, \lambda)}(\psi(t) p(t)) P_{0^{+}}^{\left(v^{\prime}, \lambda^{\prime}\right)}(\phi(t) p(t)) \\
-P_{0^{+}}^{(v, \lambda)}(\phi(t) p(t)) P_{0^{+}}^{\left(v^{\prime}, \lambda^{\prime}\right)}(\psi(t) p(t))+P_{0^{+}}^{(v, \lambda)}(p(t)) P_{0^{+}}^{\left(v^{\prime}, \lambda^{\prime}\right)}(\phi(t) \psi(t) p(t)) \mid \\
\leq\left\|\phi^{\prime}\right\|_{r}\left\|\psi^{\prime}\right\|_{s} t \int_{0}^{\frac{t}{a(1-\lambda)}} \int_{0}^{\overline{a\left(1-\lambda^{\prime}\right)}} F(t, u) G(t, v) p(u) p(v) d v d u \\
\leq\left\|\phi^{\prime}\right\|_{r}\left\|\psi^{\prime}\right\|_{s} t P_{0^{+}}^{(v, \lambda)}(p(t)) P_{0^{+}}^{\left(v^{\prime}, \lambda^{\prime}\right)}(p(t))
\end{array}
$$

This proves the result of Equation (6).

A specific case of Theorem 1 can be deduced by assuming both pathway fractional integral operators with the same parameters.

Theorem 2. Suppose $\phi(t), \psi(t) \in C_{\lambda}^{(k)}$ for $k=0$ and $v \in \mathbb{C}$, such that the real part of $v$ is positive and $\phi^{\prime} \in L_{r}([0, \infty))$ and $\psi^{\prime} \in L_{s}([0, \infty))$ with the condition $r^{-1}+s^{-1}=1$ (when $r>1$ ). $p$ is a positive function and we are choosing $a>0$ and $\lambda<1$. Thereupon for the entire $t \in[0, \infty)$ :

$$
\begin{array}{r}
\left|P_{0^{+}}^{(v, \lambda)}(\phi(t) \psi(t) p(t)) P_{0^{+}}^{(v, \lambda)}(p(t))-P_{0^{+}}^{(v, \lambda)}(\psi(t) p(t)) \cdot P_{0^{+}}^{(v, \lambda)}(\phi(t) p(t))\right| \\
\leq \frac{t}{2}\left\|\phi^{\prime}\right\|\left\|_{r}\right\| \psi^{\prime} \|_{s} \int_{0}^{\frac{t}{a(1-\lambda)}} \int_{0}^{\frac{t}{a(1-\lambda)}} F(t, u) G(t, v) p(u) p(v) d v d u \\
\leq \frac{t}{2}\left\|\phi^{\prime}\right\|_{r}\left\|\psi^{\prime}\right\|_{s}\left(P_{0^{+}}^{(v, \lambda)}(p(t))\right)^{2}
\end{array}
$$

where $P_{0^{+}}^{(v, \lambda)}(\phi(t))$ is a pathway operator in $C_{\lambda}^{(k)}$ space and $H(t, v), F(t, u)$ and $G(t, v)$ are functions in two dimensional space defined in Theorem 1. 
In the next section, we deduce some interesting and established (or new) results from Theorem 1.

\section{Derived Results}

3.1. Choose $\lambda=0$ and $a=1$

Theorem 2 will become the subsequent known inequality due to Dahmani et al. [19]:

$$
\begin{aligned}
& \left|I_{0^{+}}^{(v)}(\phi(t) \psi(t) p(t)) I_{0^{+}}^{(v)}(p(t))-I_{0^{+}}^{(v)}(\psi(t) p(t)) I_{0^{+}}^{(v)}(\phi(t) p(t))\right| \\
& \quad \leq \frac{t}{2(\Gamma(v))^{2}}\left\|\phi^{\prime}\right\|_{r}\left\|\psi^{\prime}\right\|_{s} \int_{0}^{t} \int_{0}^{t} F(t, u) G(t, v) p(u) p(v) d v d u
\end{aligned}
$$

or:

$$
\begin{aligned}
\mid I_{0^{+}}^{(v)}(\phi(t) \psi(t) p(t)) I_{0^{+}}^{(v)}(p(t)) & -I_{0^{+}}^{(v)}(\psi(t) p(t)) I_{0^{+}}^{(v)}(\phi(t) p(t)) \mid \\
& \leq \frac{t}{2}\left\|\phi^{\prime}\right\|\left\|_{r}\right\| \psi^{\prime} \|_{s}\left(I_{0^{+}}^{(v)}(p(t))\right)^{2} .
\end{aligned}
$$

Here $I_{0^{+}}^{(v)}(\phi(t))$ is a left-sided fractional integral operator of Riemann-Liouville type.

Here we note that by taking a particular value of $v$ as $v=1$, we can get the Chebyshev inequality:

$$
\begin{aligned}
\mid I_{0^{+}}^{(1)}(\phi(t) \psi(t) p(t)) I_{0^{+}}^{(1)}(p(t)) & -I_{0^{+}}^{(1)}(\psi(t) p(t)) I_{0^{+}}^{(1)}(\phi(t) p(t)) \mid \\
& \leq \frac{t}{2}\left\|\phi^{\prime}\right\|_{r}\left\|\psi^{\prime}\right\|_{s}\left(I_{0^{+}}^{(1)}(p(t))\right)^{2},
\end{aligned}
$$

that is:

$$
\begin{aligned}
\mid \int_{0}^{t} \phi(u) \psi(u) p(u) d u \int_{0}^{t} p(u) d u & -\int_{0}^{t} \psi(u) p(u) d u \int_{0}^{t} \phi(u) p(u) d u \mid \\
& \leq \frac{t}{2}\left\|\phi^{\prime}\right\|_{r}\left\|\psi^{\prime}\right\|_{s}\left(\int_{0}^{t} p(u) d u\right)^{2},
\end{aligned}
$$

for unweighted space:

$$
\left|\int_{0}^{t} \phi(u) \psi(u) d u-\int_{0}^{t} \psi(u) d u \int_{0}^{t} \phi(u) d u\right| \leq \frac{t}{2}\left\|\phi^{\prime}\right\|\left\|_{r}\right\| \psi^{\prime} \|_{s} .
$$

3.2. $\lambda$ Tends to 1 from the Left Side

From Theorem 2:

$$
\begin{aligned}
\mid \mathcal{L}_{f g p}(\xi) \mathcal{L}_{p}(\xi)- & \mathcal{L}_{g p}(\xi) \mathcal{L}_{f p}(\xi) \mid \leq \frac{1}{2 t^{2 v-1}}\left\|\phi^{\prime}\right\|\left\|_{r}\right\| \psi^{\prime} \|_{s} \\
& \times \int_{0}^{\infty} \int_{0}^{\infty} F(t, u) G(t, v) p(u) p(v) d v d u
\end{aligned}
$$

or:

$$
\left|\mathcal{L}_{f g p}(\xi) \mathcal{L}_{p}(\xi)-\mathcal{L}_{g p}(\xi) \mathcal{L}_{f p}(\xi)\right| \leq \frac{t}{2}\left\|\phi^{\prime}\right\|_{r}\left\|\psi^{\prime}\right\|_{s}\left(\mathcal{L}_{f g p}(\xi)\right)^{2}
$$

where $\xi=a v / t$ and $\mathcal{L}_{f}(\xi)$ are Laplace of the function $f$.

Since $p(t)$ is a weighted function, we can reduce Theorem 2 in unweighted form, which is given as follows: 
Corollary 1. Suppose $\phi(t), \psi(t) \in C_{\lambda}^{(k)}$ for $k=0$ and $v \in \mathbb{C}$, such that the real part of $v$ is positive and $\phi^{\prime} \in L_{r}([0, \infty))$ and $\psi^{\prime} \in L_{s}([0, \infty))$ with the condition $r^{-1}+s^{-1}=1$ (when $\left.r>1\right)$. Choose $a>0$ and $\lambda<1$. Then for whole $t \in[0, \infty)$ :

$$
\begin{array}{r}
\left|\mathcal{A} t^{v+1} P_{0^{+}}^{(v, \lambda)}(\phi(t) \psi(t))-P_{0^{+}}^{(v, \lambda)}(\psi(t)) P_{0^{+}}^{(v, \lambda)}(\phi(t))\right| \\
\leq \frac{t}{2}\left\|\phi^{\prime} \mid\right\|_{r}\left\|\psi^{\prime}\right\|_{s} \int_{0}^{\frac{t}{a(1-\lambda)}} \int_{0}^{\frac{t}{a(1-\lambda)}} F(t, u) G(t, v) d v d u \\
\leq \frac{\mathcal{A}^{2} t^{2 v+3}}{2}\left\|\phi^{\prime}\right\|_{r}\left\|\psi^{\prime}\right\|_{s^{\prime}}
\end{array}
$$

where:

$$
\mathcal{A}(\nu, \lambda)=\frac{\Gamma\left(1+\frac{v}{1-\lambda}\right)}{a(1-\lambda) \Gamma\left(2+\frac{v}{1-\lambda}\right)},
$$

which depends upon the parameter of the pathway operator.

In similar fashion, the inequality in Theorem 1 becomes:

$$
\begin{array}{r}
\mid \mathcal{B} t^{v^{\prime}+1} P_{0^{+}}^{(v, \lambda)}(\phi(t) \psi(t))-P_{0^{+}}^{(v, \lambda)}(\psi(t)) P_{0^{+}}^{\left(v^{\prime}, \lambda^{\prime}\right)}(\phi(t))-P_{0^{+}}^{(v, \lambda)}(\phi(t)) P_{0^{+}}^{\left(v^{\prime}, \lambda^{\prime}\right)}(\psi(t)) \\
+\mathcal{A} t^{(v+1)} P_{0^{+}}^{\left(v^{\prime}, \lambda^{\prime}\right)}(\phi(t) \psi(t))\left|\leq\left\|\phi^{\prime} \mid\right\|_{r}\left\|\psi^{\prime}\right\|_{s} t \int_{0}^{\frac{t}{a(1-\lambda)}} \int_{0}^{\frac{t}{a\left(1-\lambda^{\prime}\right)}} F(t, u) G(t, v) d v d u\right. \\
\leq \mathcal{A B} t^{v+v^{\prime}+3}\left\|\phi^{\prime}\right\|_{r}\left\|\psi^{\prime}\right\|_{s^{\prime}}
\end{array}
$$

where:

$$
\mathcal{B}(v, \lambda)=\frac{\Gamma\left(1+\frac{v^{\prime}}{1-\lambda^{\prime}}\right)}{a\left(1-\lambda^{\prime}\right) \Gamma\left(2+\frac{v^{\prime}}{1-\lambda^{\prime}}\right)} .
$$

Some more results can also be established by choosing $p(t)=t^{\gamma-1}$.

Corollary 2. $\phi(t), \psi(t)$ and $v$ are the same as mentioned in Theorem 2; then for all $t \in[0, \infty)$ :

$$
\begin{aligned}
& \left|\mathcal{C} t^{\nu+\gamma} P_{0^{+}}^{(v, \lambda)}\left(t^{\gamma-1} \phi(t) \psi(t)\right)-P_{0^{+}}^{(v, \lambda)}\left(t^{\gamma-1} \psi(t)\right) P_{0^{+}}^{(v, \lambda)}\left(t^{\gamma-1} \phi(t)\right)\right| \\
& \leq \frac{t}{2}\left\|\phi^{\prime}\right\|_{r}\left\|\psi^{\prime}\right\|_{s} \int_{0}^{\frac{t}{a(1-\lambda)}} \int_{0}^{\frac{t}{a(1-\lambda)}} u^{\gamma-1} v^{\gamma-1} F(t, u) G(t, v) d v d u \\
& \leq \frac{\mathcal{C}^{2} t^{2 \gamma-1}}{2}\left\|\phi^{\prime}\right\|_{r}\left\|\psi^{\prime}\right\|_{s^{\prime}}
\end{aligned}
$$

where:

$$
\mathcal{C}(\nu, \lambda, \gamma)=\frac{(\Gamma(\gamma)) \Gamma\left(1+\frac{v}{1-\lambda}\right)}{[a(1-\lambda)]^{\gamma} \Gamma\left(1+\gamma+\frac{v}{1-\lambda}\right)},
$$

which depends upon the parameter of the pathway operator as well as $\gamma$.

Similarly, the inequality of Theorem 1 reduces to:

$$
\begin{array}{r}
\mid \mathcal{D} t^{v+\gamma} P_{0^{+}}^{(v, \lambda)}\left(t^{\gamma-1} \phi(t) \psi(t)\right)-P_{0^{+}}^{(v, \lambda)}\left(t^{\gamma-1} \psi(t)\right) P_{0^{+}}^{\left(v^{\prime}, \lambda^{\prime}\right)}\left(t^{\gamma-1} \phi(t)\right) \\
-P_{0^{+}}^{(v, \lambda)}\left(t^{\gamma-1} \phi(t)\right) P_{0^{+}}^{\left(v^{\prime}, \lambda^{\prime}\right)}\left(t^{\gamma-1} \psi(t)\right)+\mathcal{C} t^{\nu+\gamma} P_{0^{+}}^{\left(v^{\prime}, \lambda^{\prime}\right)}\left(t^{\gamma-1} \phi(t) \psi(t)\right) \mid \\
\leq\left\|\phi^{\prime}\right\|\left\|_{r}\right\| \psi^{\prime} \|_{s} t \int_{0}^{\frac{t}{a(1-\lambda)}} \int_{0}^{\frac{t}{a\left(1-\lambda^{\prime}\right)}} u^{\gamma-1} v^{\gamma-1} F(t, u) G(t, v) d v d u \\
\leq \mathcal{C D} t^{v+v^{\prime}+2 \gamma+1}\left\|\phi^{\prime}\right\|\left\|_{r}\right\| \psi^{\prime} \|_{s^{\prime}}
\end{array}
$$


where:

$$
\mathcal{D}\left(v^{\prime}, \lambda^{\prime}, \gamma\right)=\frac{(\Gamma(\gamma)) \Gamma\left(1+\frac{v^{\prime}}{1-\lambda^{\prime}}\right)}{\left[a\left(1-\lambda^{\prime}\right)\right]^{\gamma} \Gamma\left(1+\gamma+\frac{v^{\prime}}{1-\lambda^{\prime}}\right)},
$$

and $t \in[0, \infty)$.

\section{Discussion and Conclusions}

We have proved two theorems in this paper, one involving pathway fractional integral operators with different parameters and the other pathway fractional integral operators with the same parameters. These two theorems are analogous versions of the Chebyshev inequality. Further, we have taken some particular cases of these theorems, choosing specific values of the parameters. Since the pathway operator is a unification of the distinct nature of operators, we can discover a number of inequalities by selecting the values applicable to the restrictions and the weighted function $p(t)$. Two of them have been shown here, viz., the Laplace operator and the left-sided Riemann-Liouville operator. Following similar methodology, we can also generalize other inequalities of the literature using pathway fractional integral operators.

Author Contributions: Conceptualization, S.D.P., A.M.M., D.B. and F.T.; methodology, validation, S.D.P., A.M.M., D.B.; formal analysis, investigation, writing-original draft preparation, A.M.M., S.D.P., D.B. and F.T.; writing-review and editing, D.B., F.T., A.M.M., S.D.P.

Funding: This research project was supported by a grant from the "Research Center of the Center for Female Scientific and Medical Colleges", Deanship of Scientific Research, King Saud University.

Acknowledgments: The authors are thankful to the referees and the editor for their valuable remarks and comments for the improvement of the paper.

Conflicts of Interest: The authors declare that they have no conflicts of interests.

\section{References}

1. Miller, K.S.; Ross, B. An Introduction to the Fractional Calculus and Fractional Differential Equations; Wiley-Interscience, John Wiley \& Sons: New York, NY, USA, 1993.

2. Samko, S.G.; Kilbas, A.A.; Marichev, O.I. Fractional Integrals and Derivatives, Theory and Applications; Gordon and Breach: London, UK, 1993.

3. Kiryakova, V. Generalized Fractional Calculus and Applications; Pitman Res. Notes Math. Ser. 301; Longman Scientific \& Technical: Harlow, UK, 1994.

4. Baleanu, D.; Diethelm, K.; Scalas, E.; Trujillo, J.J. Fractional Calculus Models and Numerical Methods; World Scientific: Singapore, 2012.

5. Mathai, A.M. A pathway to matrix-variate gamma and normal densities. Linear Algebra Appl. 2005, 396, 317-328.

6. Mathai, A.M.; Haubold, H.J. Pathway model, superstatistics, Tsallis statistics and a generalize measure of entropy. Physica A 2007, 375, 110-122.

7. Mathai, A.M.; Haubold, H.J. On generalized distributions and path-ways. Phys. Lett. A 2008, 372, $2109-2113$.

8. Nair, S.S. Pathway fractional integration operator. Fract. Calc. Appl. Anal. 2009, 12, 237-252.

9. Nisar, K.S.; Purohit, S.D.; Abouzaid, M.S.; Qurashi, M.A.; Baleanu, D. Generalized k-Mittag-Leffler function and its composition with pathway integral operators. J. Nonlinear Sci. Appl. 2016, 9, 3519-3526.

10. Dragomir, S.S. Some integral inequalities of Gruss type. Indian J. Pure Appl. Math. 2000, 31, 397-415.

11. Agarwal, P.; Choi, J. Certain fractional integral inequalities associated with pathway fractional integral operators. Bull. Korean Math. Soc. 2016 53, 181-193.

12. Ahmadmir, N.; Ullah, R. Some inequalities of Ostrowski and Grüss type for triple integrals on time scales. Tamkang J. Math. 2011, 42, 415-426.

13. Anastassiou, G.A. Advances on Fractional Inequalities; Springer Briefs in Mathematics; Springer: New York, NY, USA, 2011. 
14. Anastassiou, G.A. Fractional representation formulae and right fractional inequalities. Math. Comput. Model. 2011, 54, 3098-3115.

15. Baleanu, D.; Purohit, S.D. Chebyshev type integral inequalities involving the fractional hypergeometric operators. Abstr. Appl. Anal. 2014, 2014, 609160.

16. Baleanu, D.; Purohit, S.D.; Prajapati, J.C. Integral inequalities involving generalized Erdèlyi-Kober fractional integral operators. Open Math. 2016, 14, 89-99.

17. Baleanu, D.; Purohit, S.D.; Ucar, F. On Gruss type integral inequality involving the Saigo's fractional integral operators. J. Comput. Anal. Appl. 2015, 19, 480-489.

18. Choi, J.; Purohit, S.D. A Gruss type integral inequality associated with Gauss hypergeometric function fractional integral operator. Commun. Korean Math. Soc. 2015, 30, 81-92.

19. Dahmani, Z.; Mechouar, O.; Brahami, S. Certain inequalities related to the Chebyshev's functional involving a Riemann-Liouville operator. Bull. Math. Anal. Appl. 2011, 3, 38-44.

20. Farid, G.; Pečarić, J.; Tomovski, Ž. Opial-type inequalities for fractional integral operator involving Mittag-Leffler function. Fract. Differ. Calc. 2015, 5 93-106.

21. Iqbal, S.; Pečarić, J.; Samraiz, M.; Tomovski, Ž. Hardy-type inequalities for generalized fractional integral operators. Tbil. Math. J. 2017, 10, 75-90.

22. Purohit, S.D.; Raina, R.K. Chebyshev type inequalities for the Saigo fractional integrals and their $q$-analogues. J. Math. Inequal. 2013, 7, 239-249.

23. Purohit, S.D.; Raina, R.K. Certain fractional integral inequalities involving the Gauss hypergeometric function. Rev. Téc. Ing. Univ. Zulia 2014, 37, 167-175.

24. Saxena, R.K.; Purohit, S.D.; Kumar, D. Integral inequalities associated with Gauss hypergeometric function fractional integral operator, Proc. Nat. Acad. Sci. India Sect. A Phys. Sci. 2018, 88, 27-31.

(C) 2019 by the authors. Licensee MDPI, Basel, Switzerland. This article is an open access article distributed under the terms and conditions of the Creative Commons Attribution (CC BY) license (http:/ / creativecommons.org/licenses/by/4.0/). 